\title{
Recursos da web 2.0 no mestrado em ciências da educação no instituto superior de ciências de educação do Huambo
}

\author{
Los recursos de la web 2.0 en la Maestría en Ciencias de la Educación \\ en el Instituto Superior de Huambo
}

The resources of web 2.0 in the master's degree of educations sciences at the Higher Institute of Education Sciences of Huambo

\section{João Baptista Machado Sousa* y Georgina Díaz Fernández**}

\section{RESUMO}

O surgimento da web 2.0 e seus recursos, no final de 2004, causaram uma grande mudança de paradigma no sector da educação; tendo em conta que deixamos de ser meros espectadores e passamos a ser protagonistas das actividades que se realizam na web, favorecendo, desta forma, a criação e publicação de conteúdos na rede; a interacção de forma síncrona e o trabalho de forma colaborativa. O presente artigo aborda a experiência da utilização de alguns destes recursos (plataforma edmodo, ferramenta colaborativa google drive, blogs e o gestor de referências bibliográficas mendeley) no mestrado em ciências de educação e educação em conservação da natureza, no Instituto Superior de Ciências de Educação do Huambo, República de Angola. Trabalhou-se com uma população de 80 estudantes onde se desenhou, implementou e se avaliou um curso, utilizando estas ferramentas. Para levar a cabo o processo de formação, estabeleceram-se três etapas: a primeira faz referência à seleção da equipa de trabalho e dos recursos da web 2.0; a segunda, ao desenho e implementação do curso; e a terceira refere-se à avaliação do curso. Tiveram-se em conta três princípios: princípio do ensino e da aprendizagem flexível, interactivo e colaborativo, princípio da unidade entre instrução, educação e desenvolvimento e, finalmente, o princípio do vínculo entre a teoria e a prática. No final da formação, a população alvo de investigação demonstrou estar satisfeita mediante o questionário aplicado onde reconhece que os resultados da formação foram acima das expectativas.

\footnotetext{
* Doutor em Ciências Pedagógicas, Universidade de Ciências Pedagógicas "Enrique José Varona" - Cuba. Professor e investigador do Instituto Superior de Ciências de Educação de Huambo. Huambo, Angola. Correio: sousangola@gmail.com

** Doutora em Ciências Pedagógicas, Universidade de Ciências Pedagógicas "Enrique José Varona" - Cuba. Professora e investigadora da mesma Universidade. Havana, Cuba. Correio: georginadf@ucoejv.edu.cu
}

82 
João Baptista Machado Sousa y Georgina Díaz Fernández

Telos Vol. 20, No. 1 (2018). 82-100

Palavras-Chave: Recursos Web 2.0, Mestrado, ISCED-Huambo

RECIBIDO: Septiembre 2017 ACEPTADO: Noviembre 2017

\begin{abstract}
The emergence of web 2.0 and its resources at the end of 2004 caused a great paradigm shift in the education sector, taking into account that we are no longer mere spectators and we become protagonists of the activities that take place on the web, favoring in this way the creation And publication of content on the network, interaction synchronously and work collaboratively. This article discusses the experience of using some of these resources (edmodo platform, google drive collaborative tool, blogs and bibliographic reference manager medley) in the master's degree in education sciences and education in nature conservation, at the Higher Institute of Educational Sciences Of Huambo, Republic of Angola. We worked with a population of 80 students where a course was designed, implemented and evaluated using these tools. In order to carry out the training process, three steps were established: the first refers to the selection of the work team and web 2.0 resources; the second, to the design and implementation of the course; And the third relates to course evaluation. Three principals have been taken into account: principle of teaching and flexible, interactive and collaborative learning, principle of unity between education, education and development and finally the principle of the link between theory and the practice. At the end of the training the target population of research showed that they were satisfied using the questionnaire applied where they recognize that the training results were above expectations.
\end{abstract}

Keywords: Web 2.0 Resources, Master Degree, ISCED-Huambo.

\title{
Introdução
}

Nos últimos dez anos, o tema "Recursos da Web 2.0" tem sido dos mais recorrentes na internet; aglomeram-se resultados que vão desde reflexões, investigações e experiências até o uso de diferentes ferramentas colaborativas que varia entre o ensino fundamental ao universitário.

Em Angola, existem experiências sistematizadas de Instituições de Ensino Superior que utilizam estes recursos no processo de formação profissional. Entre os autores que têm realizado significativas contribuições, destacam-se: Jamba (2008) que desenhou uma plataforma educativa para a formação à distância na Faculdade de 83 
Recursos da web 2.0 no mestrado em ciências da educação no instituto superior de ciências de educação do Huambo

Ciências da Universidade Agostinho Neto; Samy (2014) que propõe um modelo de virtualização dos processos substantivos no Instituto Superior de Ciências de Educação em Luanda; Sousa (2015) que desenhou uma concepção teóricometodológica para a superação dos professores do Instituto Superior de Ciências de Educação do Huambo em ambiente virtual de ensino-aprendizagem; Da Costa (2016), que propõe um modelo de ensino à distância sustentável em Angola.

Nesta perspectiva, o Instituto Superior de Ciências de Educação do Huambo, mais adiante ISCED-Huambo, começou a experimentar algumas destas ferramentas nas formações ao nível de pós-graduação profissional, realizados em 2012, 2013 e 2014 com resultados satisfatórios, como os abordados nos artigos de autores como: Robalo e Gouveia (2013), Sousa e Díaz (2013), apresentado no XIII congresso internacional "Pedagogia 2013" em Cuba.

Em Abril de 2017, o ISCED-Huambo lançou a sua primeira edição de pósgraduação acadêmica (mestrado) para o curso de Ciências de Educação, no qual utilizou-se alguns dos recursos da web 2.0 no módulo de informática educativa com o objetivo de favorecer o trabalho colaborativo e facilitar a realização das actividades de trabalho independente.

Neste artigo, abordam-se experiências sistematizadas sobre a utilização destas ferramentas no mestrado em ciências de educação em realização no ISCEDHuambo, Angola.

\section{Recursos da web 2.0 e seu impacto na educação.}

Antes de abordar sobre os recursos da web 2.0 e seu impacto na educação, seria interessante realizar uma reflexão sobre o conceito de web 2.0. Nesta direcção, a sistematização teórica realizada à volta deste tema, permitiu identificar que existe consenso em que este termo foi usado pela primeira vez por Tim O'Reilly e Dale Dougherty, durante uma sessão de "brainstorming" realizada em 2004, onde 
João Baptista Machado Sousa y Georgina Díaz Fernández.

Telos Vol. 20, No. 1 (2018). 82-100

examinavam a situação que vivia a web nos anos de 2001 (Garrido, S/D; O’Reilly, 2005; Bottentuit, Iahn e Bentes, 2008; Moreira e Dias, 2009).

Daí em diante, a concepção 2.0 atingiu o seu auge até que em 2005 Tim O'Reilly, escreveu um artigo intitulado "What is Web 2.0" no qual esclarece o significado deste termo, uma vez que por um lado existia algumas críticas por parte da comunidade científica, e por outro aceitavam como um novo paradigma.

A web 2.0 é a segunda geração dos serviços disponíveis na internet, faz referência ao uso destas ferramentas de forma produtiva, ou seja, com recursos que permitem aos usuários, não só buscar por informações, mas também serem productores de conteúdos na rede.

Assim é que quando aborda-se os recursos da web 2.0, está-se a fazer referência a um conjunto de serviços que facilitam a comunicação, o armazenamento, a partilha e a transferência de informação, a busca, a cooperação e a colaboração.

No que diz respeito à sua classificação, Cobo e Pardo (2007), consideram que podem ser estruturados em: redes sociais, conteúdos, organização social e inteligente da informação e aplicações e serviços.

Na visão de Manuel Área, agrupa-os em: publicação de espaços pessoais (blogs), publicação compartilhada (wikis), publicação de apresentações multimedia (Slideshare), publicação de arquivos de imagem (flickr, picasa), publicação de arquivos audiovisuais (Youtube, Google Vídeo), etc”. (Área, 2009, p. 71).

Não obstante, Leite e Leão (2009) afirmam que os recursos da Web 2.0 podem ser classificados em duas categorias:

- Na primeira: incluem-se as aplicações que só podem existir na Internet, cuja eficácia aumenta com o número de utilizadores registrados, como por exemplo: google docs \& spreadsheets, wikipédia, del.icio.us, youtube, skype, ebay, hi5, etc. 
Recursos da web 2.0 no mestrado em ciências da educação no instituto superior de ciências de educação do Huambo

- Na segunda: incluem-se as aplicações que podem funcionar offline, mas que também podem trazer grandes vantagens se estiverem online: picasa fotos, google maps, mapquest, itunes, etc.

Ardila (2009), considera que os recursos da web 2.0 podem ser classificados como: ferramentas de repositório, ferramentas de integração e ferramentas de produção.

Por outro lado, o monográfico Scopeo, na sua primeira edição, classifica como sendo: redes sociais, criação de recursos, recuperação de informação e partilha de recursos. (Scopeo, 2009).

Verifica-se desta forma, que apesar da divergência de critérios, alguns autores coincidem quando abordam sobre a partilha de informação como uma das vias para classificar a web 2.0 (Área, 2009; Scopeo, 2009), outros fundamentam os espaços pessoais ou redes sociais como um elemento a ter-se em conta na sua classificação. (Cobo e Pardo, 2007; Scopeo, 2009).

$\mathrm{Na}$ perspectiva dos autores desta investigação e tendo em conta a intencionalidade deste artigo, agrupou-se em:

- Recursos para comunicação: skype, viber, whats app, twitter, facebook, podcast;

- Recursos para armazenamento: dropbox, onedrive, googledrive;

- Recursos para partilha e transferência de informação: picassa, flicker, google drive, slideshare, dropbox, onedrive, delicious;

- Recursos para cooperação e colaboração: edmodo, moodle, google drive, dropbox, blogs, wikis, joomla, só para citar alguns. ${ }^{1}$

\section{Impacto da web 2.0 na educação.}

\footnotetext{
${ }^{1}$ Algumas destas ferramentas podem realizar as quatro funções (comunicação, armazenamento, partilha e colaboração).
} 
João Baptista Machado Sousa y Georgina Díaz Fernández Telos Vol. 20, No. 1 (2018). 82-100

A web 2.0 e seus recursos têm grande impacto na educação, uma vez que com o seu surgimento, multiplicaram-se e modernizaram-se os ambientes utilizados para a construção do conhecimento. Nesta direcção, Marqués (2007), ao se pronunciar sobre o uso da web 2.0 na educação, afirma que:

- Constituem um espaço social horizontal e rico em fontes de informação (rede social onde o conhecimento não está fechado), que representa uma alternativa à tradicional hierarquia e unidirecionalidade da aprendizagem. Implica novos papéis para professores e alunos orientados ao trabalho autónomo e colaborativo, crítico e criativo, à expressão pessoal, a investigar e a partilhar recursos, a criar conhecimento e a aprender;

- As suas fontes de informação (embora nem todas fiáveis) e canais de comunicação facilitam a aprendizagem mais autónoma e permitem uma maior participação nas actividades de grupo, o que aumenta o interesse e a motivação dos alunos;

- Com as suas aplicações de edição, professores e alunos podem elaborar facilmente materiais individualmente ou em grupo, partilhá-los e submetê-los aos comentários dos leitores;

- Proporciona espaços online para o armazenamento, classificação e publicação/difusão de conteúdos textuais e audiovisuais, onde todos possam aceder;

- Facilita a realização de novas actividades de aprendizagem e de avaliação e a criação de redes de aprendizagem;

- Desenvolve e melhora as competências digitais, desde a procura e selecção de informação e correspondente processo para a converter em conhecimento, até a sua publicação e transmissão em diferentes suportes;

- Proporciona ambiente para o desenvolvimento de redes de escolas e professores para reflectir sobre os temas educativos, para ajudar, elaborar e 
Recursos da web 2.0 no mestrado em ciências da educação no instituto superior de ciências de educação do Huambo

partilhar recursos. Portanto, quando falamos de Web 2.0 e Educação referimo-nos a uma abordagem metodológica de aprendizagem que favorece a construção de conhecimento com base na participação colectiva do grupo.

- O uso pedagógico de ferramentas Web 2.0 proporciona a implementação de modelos pedagógicos baseados na interacção, na colaboração, na inovação e na promoção da autonomia do aluno.

Por outro lado, De Haro (2008), ao abordar sobre a utilização destes recursos, destaca três áreas prioritárias: comunicativa, publicação de conteúdos e a de recuperação de informação.

Não obstante, Scopeo (2009), aborda quatro vias para a utilização dos recursos da web 2.0 na educação, sendo:

- Software social para compartilhar recursos;

- Software social para recuperar informação;

- Software social para desenvolver recursos;

- Software social para criar redes sociais.

Hugo Emílio Traverso e colaboradores, quando se dirigem sobre a web 2.0 e seu impacto na educação, afirmam que se poderia falar de uma "aprendizagem 2.0" que se apoia em dois principios básicos: conteúdos gerados pelos usuários e arquitectura da participação. A ideia principal desta aprendizagem é alcançar um conhecimento intercambiável, acumulativo, colaborativo, que pode ser partilhado, transferido e convertido em um bem público (Traverso e outros, 2013).

Os mesmos autores consideram que existem quatro tipologias diferentes de aprendizagem 2.0:

a) Aprender fazendo: Para este tipo de aprendizagem utilizam-se ferramentas que permitem ao estudante e/ou docente a escritura e leitura na Web, sob o princípio de "ensaio-erro". Por exemplo, os estudantes podem aprender sobre biologia, gerando 
João Baptista Machado Sousa y Georgina Díaz Fernández. Telos Vol. 20, No. 1 (2018). 82-100

apresentações em linha sobre diferentes tópicos do tema. Logo, o professor revisa e corrige aqueles aspectos melhoráveis gerando uma apresentação mais completa.

b) Aprender interactuando: Este enfoque apoia-se no intercâmbio de conhecimentos através de ferramentas online como chats, foros e correios eletrônicos. Por exemplo, o docente poderia expor uma temática que os alunos devem debater por chat, fora do horário de aula.

c) Aprender procurando: Este tipo de aprendizagem consiste na busca de fontes que oferecem informação sobre um tema determinado. Este processo de investigação, selecção e adaptação terminam ampliando e enriquecendo o conhecimento de quem o realiza.

d) Aprender compartilhando: $\mathrm{O}$ processo de intercâmbio de conhecimentos e experiências permite aos estudantes participar activamente de uma aprendizagem colaborativa. A internet conta com uma grande quantidade de recursos para que os alunos compartilhem os conteúdos que eles mesmos produziram.

Verifica-se desta forma, diferentes vias para fundamentar o impacto que a web $2.0 \mathrm{e}$ seus recursos exercem sobre a educação, apesar da divergência de critérios, os autores consultados são unânimes em afirmar que a web 2.0 e seus recursos quando bem aproveitados, podem ser um factor fundamental na mudança de paradigma no setor da educação, promovendo a aparição de modernos ambientes virtuais de aprendizagem e favorecendo o trabalho colaborativo.

\section{Metodologia e resultados}

Para levar a cabo o processo de formação dos mestrandos no ISCEDHuambo e a utilização dos recursos da web 2.0, estabeleceu-se três etapas: a primeira faz referência à seleção da equipa de trabalho e dos recursos da web 2.0; a segunda, ao desenho e implementação do curso; e a terceira refere-se à avaliação do curso. 
Recursos da web 2.0 no mestrado em ciências da educação no instituto superior de ciências de educação do Huambo

\section{Etapa 1. Seleção da equipa de trabalho e dos recursos da web 2.0}

Nesta etapa, avaliou-se a importância do curso, as necessidades do mesmo, a quem vai dirigido, as possibilidades de o realizar, formalizou-se de maneira oficial, mediante os documentos, as acções que se realizaram e selecionou-se o grupo de trabalho. Durante esta fase, precisou-se o problema educativo a resolver, identificouse sua fonte e determinou-se as possíveis soluções. Além disso, procurou-se entender os objectivos, as metas, capacidades e motivações dos possíveis estudantes. Para tal:

- Selecionou-se o grupo de trabalho (docentes e estudantes);

- Analisou-se as tarefas necessárias para elaborar o curso;

- Caracterizou-se o curso e os possíveis estudantes;

- Realizou-se uma análise da disponibilidade tecnológica.

Nesta perspectiva, a seleção dos professores para participar nesta formação foi feita em função dos seguintes indicadores:

- Formação acadêmica e científica que possuem;

- Produção científica que acumulam;

- Disciplina que leccionam na graduação;

- Experiência que possuem em realizar formação com recursos da web 2.0.

Por outro lado, os mestrandos foram selecionados em função dos seguintes indicadores:

- Média final de graduação;

- Função que desempenham;

- Produção científica que acumulam;

- Motivação que apresentam para frequentar a formação.

Como resultado desta análise, selecionou-se uma equipa de trabalho composta por dois professores doutores com experiência na utilização de recursos da web 2.0 e oitenta estudantes. Os mesmos têm formação de base heterogénea, entre 
João Baptista Machado Sousa y Georgina Díaz Fernández

Telos Vol. 20, No. 1 (2018). 82-100

licenciados no ensino da matemática, pedagogia, psicologia, geografia, biologia há engenheiros agrônomos como nos mostra o gráfico abaixo.

Fig. I. Gráfico sobre a formação de base dos mestrandos

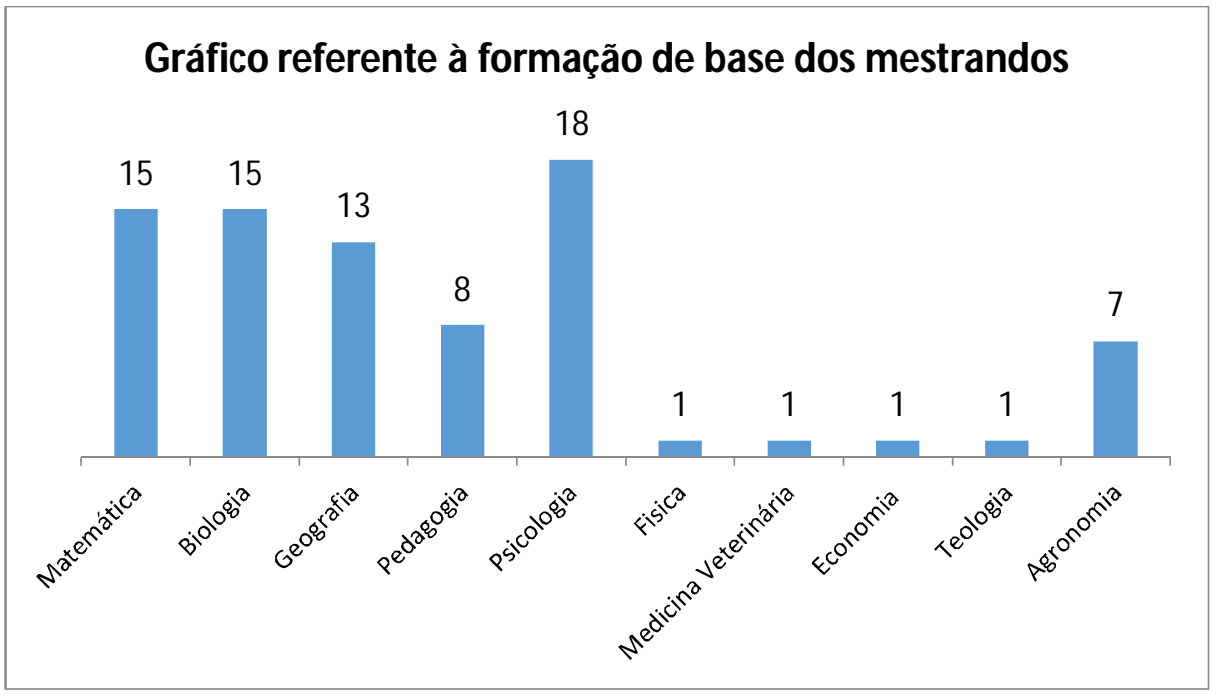

Fonte: elaboração própria

Nesta direcção, depois de sistematizados os referentes teóricos e metodológicos que abordam sobre estes conteúdos, analisadas as experiências das formações passadas, das necessidades de formação e tendo em conta a função laboral que os mestrandos desempenham, selecionaram-se os seguintes recursos:

- Edmodo: é uma rede social educativa, livre, na qual cada educador tem a possibilidade de se cadastrar, criar grupos de trabalhos e usufruir das ferramentas que a mesma dispõe. É um ambiente virtual, no qual a sua filosofia de ensino é baseada no conectivismo por ser uma rede social educativa. Sousa (2017). Esta ferramenta foi selecionada e utilizada tendo em conta o enfoque construtivista do processo de ensino-aprendizagem, com a finalidade de criar um entorno virtual 
Recursos da web 2.0 no mestrado em ciências da educação no instituto superior de ciências de educação do Huambo

colaborativo, onde os mestrandos pudessem ter acesso a toda a bibliografia, as actividades de aprendizagem, realizar avaliações, co-avaliações, auto-avaliações, nos formatos de questionários e tarefas que implicavam a investigação de diferentes temas e, finalmente, o fórum onde se debatiam algumas temáticas que eram abordados em sala de aulas.

- Google Drive: é um recurso da Web 2.0, que, anteriormente, era conhecido como Google Docs. Actualmente, oferece diferentes serviços como o de armazenamento de arquivos na núvem e sincronização destes com outros dispositivos. Esta ferramenta da web 2.0 foi selecionada e utilizada com a finalidade de se criar, compartilhar e trabalhar de forma colaborativa em diferentes materiais, que eram elaborados em sala de aulas. Para além de partilhar o relatório de todas as actividades realizadas entre o professor e os estudantes e a criação dos instrumentos de recolha de dados com o formulário da google, que foi utilizado para avaliar o estado de ânimo, a satisfação e o seu desejo em continuar a trabalhar com estas ferramentas.

- Blogs: Este termo foi criado em finais da década de 1990, precisamente em 1997 por Jorn Barger. Esta palavra refere-se a um diário na Web, cuja informação está organizada da mais recente para a mais antiga, frequentemente actualizado com opiniões, emoções, factos, imagens para além de disponibilizar um índice de entrada e pode conter hiperligação para outros sites. A seleção desta ferramenta permitiu aos mestrandos aprenderem a criar os seus blogs pessoais, blogs para as disciplinas que lecionam e para a actividade profissional que exercem. Os mestrandos foram orientados a publicar todas as informações relacionadas as actividades que realizam no seu quotidiano.

- Mendeley: é uma rede social académica, que permite realizar a gestão integral de documentos, fundamentalmente do tipo PDF de forma ubíqua, para além de gerir citações e bibliografia. Sousa (2017). A seleção desta ferramenta permitiu aos 
João Baptista Machado Sousa y Georgina Díaz Fernández.

Telos Vol. 20, No. 1 (2018). 82-100

mestrandos aprender a gerir de forma integral, toda a informação para posterior utilização nos trabalhos científicos, que deverão realizar. Para além da gestão da informação, aprenderam a criar grupos de trabalho, a partir do mendeley web e trabalhar de forma colaborativa nesta mesma ferramenta.

\section{Etapa 2. Desenho e implementação do curso}

O desenho é o momento em que se especifica como se levará a cabo o processo de ensino-aprendizagem. Neste momento, desenhou-se o curso em correspondência com as necessidades dos estudantes, para o qual as actividades anteriores são fundamentais. Implicou pôr em prática as estratégias para desenvolver o processo de ensino-aprendizagem. A implementação é a etapa em que se revisa e implementa o curso. O propósito deste momento é o desenvolvimento efectivo do curso. Revisou-se para determinar a sua adequação com os objectivos anteriormente planificados e pôr-se em prática a experiência educativa. Para tal foi necessário:

- Procurar e processar a informação científica sobre a temática em consonância com os objectivos estabelecidos;

- Determinar os recursos necessários e desenhar a estrutura do curso;

- Elaborar o guia de estudos;

- Elaborar os conteúdos, tarefas, os princípios norteadores e a bibliografia;

- Determinar as estratégias de ensino;

- Revisar o curso elaborado;

- Implementar o curso.

O guia de estudo, neste módulo, foi utilizado como ferramenta para orientar os estudantes nas diferentes actividades que se propuseram a realizar. Para este curso o guia foi desenhado em função dos objectivos que figuram no programa de estudos, com orientações precisas das actividades que se devem desenvolver. 
Recursos da web 2.0 no mestrado em ciências da educação no instituto superior de ciências de educação do Huambo

Por outro lado, foram desenhadas oito actividades de ensino-aprendizagem, entre questionários de auto-avaliação, tarefas onde os estudantes tinham de investigar diferentes temas e utilizar outros recursos da web para além de enviar o resultado da pesquisa pela plataforma; e, finalmente, o fórum obrigatório onde cada mestrando devia dar o seu contributo a volta do tema que estivesse a ser abordado.

Para o desenvolvimento deste curso, tiveram-se em conta os seguintes princípios:

\section{Princípio do ensino e da aprendizagem flexível, interactivo e} colaborativo: este princípio permite acomodar-se diretamente às formas em que se ensina e se aprende, os diferentes níveis de aprendizagem, diferentes necessidades e lugares de aprendizagem, as opções e graus de controlo ao estudante. A interação facilita o processo de construção do conhecimento, permite o feedback entre as actividades de aprendizagem e o estudante e é um elemento a ter em conta no momento do desenvolvimento de um curso. $\mathrm{O}$ trabalho colaborativo, entendido como aquele processo que faz potencializar os esforços do grupo entre os professores e os estudantes, e que requer participação activa e interação por parte de ambos, professores e estudantes, frente aos modelos tradicionais de aprendizagem acumulativa. Permite aos estudantes trabalharem com outros para alcançar objetivos em comum.

Princípio da unidade entre instrução, educação e desenvolvimento: a instrução expressa o resultado da assimilação de conhecimentos, hábitos e habilidades. Caracteriza-se pelo nível de desenvolvimento do intelecto e das capacidades criadoras do homem. Pressupõe determinado nível de preparação do indivíduo para sua participação nas diferentes esferas da vida social. Enquanto a educação é o comportamento adequado segundo as exigências de uma sociedade dada, em um momento histórico determinado. Esse comportamento adequado exige certa instrução e, por sua vez, é muito improvável que se possa obter uma instrução acabada, sem um comportamento segundo as exigências da sociedade. Esta relação 
João Baptista Machado Sousa y Georgina Díaz Fernández.

Telos Vol. 20, No. 1 (2018). 82-100

não se pode ver de maneira estática, imóvel, deve entender-se de forma activa, interactuando entre os seus elementos, não só na interação educação-instrução, mas também entre aqueles e o meio social. Tal como se expôs, a sociedade lhe expõe seu encargo social à escola, encargo que esta procura satisfazer, e o formado, produto da escola e do meio, em ocasiões actua sobre o meio e sobre a ciência, o que repercute sobre a escola e desta forma se produz a evolução social e escolar.

Princípio do vínculo entre a teoria e a prática: este princípio garante que os estudantes não somente se apropriem de um sistema lógico de conhecimentos, mas também que se apliquem na prática para resolver as necessidades da produção e os serviços em geral e em particular para contribuir na solução do problema identificado nos seus locais de trabalho. As aulas têm uma parte teórica onde os mestrandos apreciam o fenômeno ou objecto de estudo em seu próprio meio nas condições originais; e outra prática onde, mediante todos os conhecimentos teóricos obtidos os mestrandos são orientados a realizar diferentes actividades práticas. A sua essência levou alguns pedagogos a denomina - lo como princípio de vinculação da escola com a vida. Deve-se esclarecer que considera - lo constitui uma das exigências do princípio do carácter científico e educativo do ensino, já que só quando os estudantes podem utilizar os conhecimentos para resolver os problemas da vida, aumenta neles o valor da ciência e educam-se conscientemente.

\section{Etapa 3. Avaliação da formação}

Ocorre ao longo de todo o processo, dentro das etapas, entre as fases, e depois da implementação. Centra-se na efectividade global da experiência educativa. Além disso deve-se recolher opiniões dos estudantes para possíveis melhorias e actualizações do curso. Para tal deve-se:

- Determinar os critérios de avaliação;

- Avaliar os resultados da formação;

- Actualizar os recursos utilizados durante a formação. 
Recursos da web 2.0 no mestrado em ciências da educação no instituto superior de ciências de educação do Huambo

Para avaliar os resultados da utilização dos recursos selecionados, estabeleceram-se os seguintes momentos:

a) Determinação dos critérios de avaliação: neste momento determinou-se os aspectos a serem avaliados. Como avaliar? Que instrumento utilizar para avaliação? Que via utilizar? Que tipo de perguntas deve constar nos instrumentos? Depois de analisado todos os aspectos antes referenciados, desenhou-se um inquérito com a ferramenta formulário da google, e nela constaram perguntas do tipo: abertas, fechadas e semi abertas.

b) Avaliação dos resultados da formação: depois de desenhado o inquérito com o formulário do Google, partilhou-se com os estudantes para avaliar a formação no sentido amplo e em particular a utilização dos recursos da web 2.0. A análise deste instrumento nos remete ao seguinte gráfico:

Fig. II. Gráfico sobre o nível de satisfação dos mestrandos pela formação

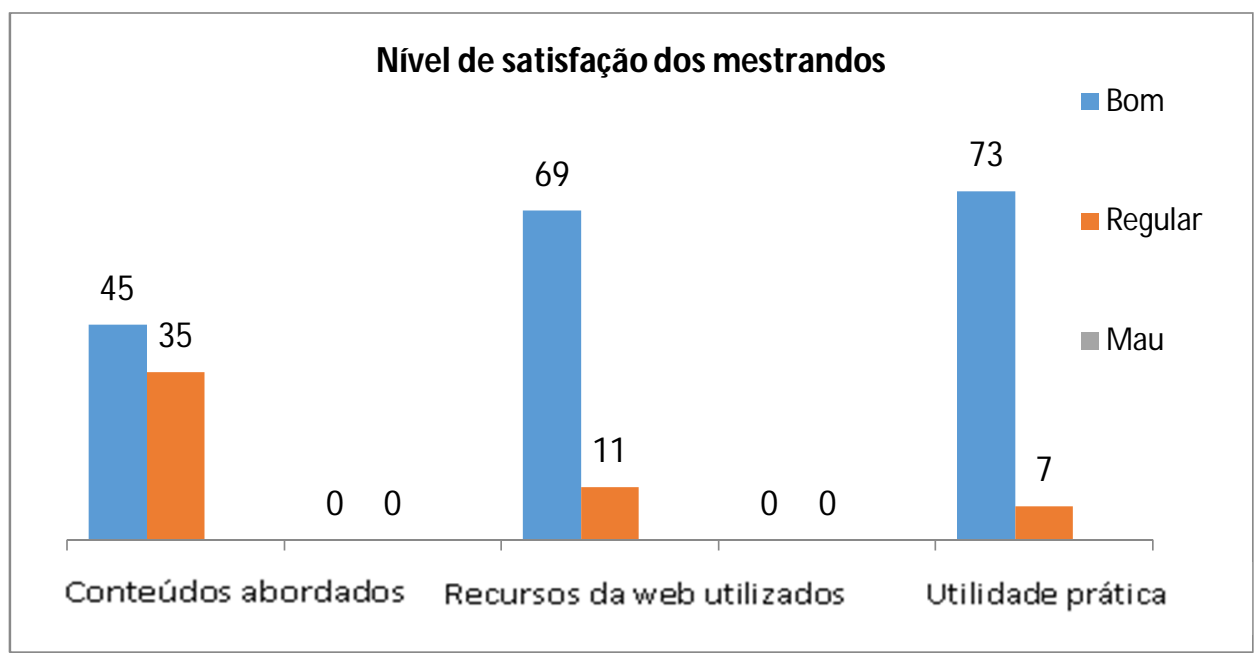

Fonte: elaboração própria 
João Baptista Machado Sousa y Georgina Díaz Fernández.

Telos Vol. 20, No. 1 (2018). 82-100

c) Actualização dos recursos utilizados durante a formação: O resultado do inquérito aplicado aos estudantes mostra que na sua maioria estão satisfeitos com os recursos da web 2.0 selecionados.

Estes resultados indicam que a utilização de um ambiente virtual, favoreceu a comunicação entre os elementos do grupo e entre o grupo e o professor, permitiu ainda a realização de actividades de ensino-aprendizagem e das avaliações.

Nesta direcção utilizou-se blogs fundamentalmente para a publicação de conteúdos na rede, e a internet, facilitou o desenvolvimento e a melhoria das competências digitais, que se manifestam na procura, seleção e recuperção de informação, utilizando diferentes buscadores e técnicas de pesquisa. De forma geral os estudantes afirmam que foi uma experiência que proporcionou a interação com diferentes fontes de informação e canais de comunicação e facilitou a aprendizagem mais autónoma para além de que permitiu uma maior participação nas actividades de grupo, o que elevou o interesse e a motivação dos mestrandos pela formação, concordando desta forma com as teorias de Marqués (2007) e De Haro (2010).

Não obstante a estes resultados solicitam a possibilidade de integrar alguns recursos que permitam desenhar cronologia digital como o timeline, a edição de vídeos com software como o movie maker ou camtasia e a publicação dos mesmos na youtube. Estas contribuições serão tidas em conta no momento da actualização dos recursos para o próximo ciclo de formação.

\section{Considerações finais}

Como resultado da sistematização teórica realizada nos últimos dois anos em torno dos recursos da web 2.0 e seu impacto na educação, apresentam-se as seguintes considerações: 
Recursos da web 2.0 no mestrado em ciências da educação no instituto superior de ciências de educação do Huambo

As transformações que ocorrem ao nível do mundo como resultado dos avanços da ciência e da tecnologia, demonstram a necessidade de implementação de alguns dos recursos da web 2.0 na educação, tendo em conta que favorecem a preparação e actualização de quem as utiliza e contribui a fomentar o interesse pelas formações que se realizam nas diferentes modalidades de ensino.

O desenho do módulo de Informática Educativa no mestrado em ciências de educação no ISCED-Huambo, obedeceu a três etapas, sendo a seleção da equipa de trabalho e dos recursos da web 2.0, o desenho e implementação do curso e finalmente a avaliação da formação, o qual demostrou resultados satisfatórios.

A experiência de utilização destes recursos no ciclo de formação ora mencionado demonstrou por um lado, as vantagens da utilização destas ferramentas tendo em conta que favorecem a realização de trabalhos de forma colaborativa e contribuem para uma aprendizagem mais construtivista. Por outro lado, apesar da avaliação positiva feita pelo grupo de trabalho, os instrumentos aplicados para recolha de dados na etapa da avaliação da formação, demostraram a necessidade de um processo de actualização, aspecto que será considerado no próximo ciclo de formação.

\section{Referências Bibliográficas}

Ardila, Patricia. (2009). Que es la web 2.0 y clasificación de sus herramientas. Disponível em: http://www.authorstream.com/Presentation/pattiardila1903834-que-es-la-web-clasificacion-de-sus-herramientas. Consulta: $05 / 07 / 2017$.

Area, Moreira, Manuel. (2009). Introducción a la Tecnología Educativa. Disponível em: http://manarea.webs.ull.es/wp-content/uploads/2010/06/ebookte.pdf Consulta: 10/9/2015.

Bottentuit, João Baptista; Iahn, Luciene Ferreira; Bentes, Roberto de Fino. (2008). As ferramentas da Web. 2.0 nas organizações: vantagens e contextos de utilização. 
João Baptista Machado Sousa y Georgina Díaz Fernández

Telos Vol. 20, No. 1 (2018). 82-100

http://publica.fesppr.br/index.php/rnti/issue/download/5/38. Consulta: 12/11/2015.

Cobo, Christóbal e Pardo, Hugo. (2007). Planeta Web 2.0. Inteligencia colectiva o medios fast food. Barcelona / México DF: Grup de Recerca d'Interaccions Digitals, Universitat de Vic / Flacso México. Disponível em: http://www.planetaWeb2.net/ Consulta: 02/08/2017.

Da Costa, Venâncio Ferreira. (2016). Modelo de ensino a distancia sustentável em Angola. Tese doutoral. La Habana. Universidad de La Habana. Cuba.

De Haro, Juan José. (2010). Educativa. Disponível em: http://jjdeharo.blogspot.com/. Consultado: 04/06/2015.

Garrido, Carlos Castaño. (S/D). Educar con redes sociales y web 2.0. Disponível em: http://servicio.bc.uc.edu.ve/educacion/eduweb/vol2n2/art2.pdf Consulta: $17 / 10 / 2017$

Jamba, Manuel. (2008). Metodología para la enseñanza desarrolladora del Álgebra Lineal, en la modalidad a Distancia, con el uso de las Tecnologías de la Información y Comunicación para la Carrera de economía de la Universidad Agostinho Neto de la República de Angola. Tese doutoral. La Habana. UCPEJV. Cuba.

Leite, Bruno Silva; Leão e Marcelo Brito Carneiro. (2009). A Web 2.0 como ferramenta de aprendizagem no ensino de ciências. UFR de P. Recife. Brasil. Disponível em: http://www.tise.cl/2009/tise_2009/pdf/10.pdf. Consulta: 10/06/2017

Marqués, Pere. (2007). La Web 2.0 y sus aplicaciones didácticas. Disponível em: http://www.peremarques.net/web20.htm. Consulta: 18/04/2013.

Moreira, Danilo dos Reis e Dias, Márcio de Souza. (2009). Web 2.0 - a web social.

Revista CEPPG. Volume. 11. No 20. Brasil. (Pp. 196-208).

O'reilly, Tim. (2005). What is Web 2.0. Design Patterns and Business Models for the Next Generation of Software. Disponível em: http://www.oreillynet.com/pub/a/oreilly/tim/news/2005/09/30/what-isweb-20.html?page=1. Consulta: 10/08/2009.

Robalo, Ana Alexandre Pereira e Gouveia, Luis Borges. (2013). As tecnologias na educação: um novo olhar pedagógico no ambiente virtual edmodo. XIII Congreso Internacional de Pedagogía. La Habana, Cuba.

Samy, José. (2014). Modelo de virtualización de la formación en el Instituto Superior de Ciencias de la Educación de Luanda. Tese doutoral. La Habana. IPLAC. Cuba.

Scopeo. (2009). Formación Web 2.0. Monográfico SCOPEO. № 1 . Disponível em: http://scopeo.usal.es/wp-content/uploads/2012/10/scopeom001.pdf. Consulta: 03/07/2017

Sousa, João Baptista Machado. (2015). La superación profesional de profesores del Instituto Superior de Ciencias de la Educación de Huambo, en 
Recursos da web 2.0 no mestrado em ciências da educação no instituto superior de ciências de educação do Huambo

ambientes virtuales de enseñanza-aprendizaje. Tesis doctoral. La Habana, UCPEJV. Cuba.

Sousa, João Baptista Machado. (2017). Informática Educativa. Novas Edições Acadêmicas. Alemanha

Sousa, João Baptista Machado e Díaz, Georgina. (2013). Experiencia didáctica con el Edmodo en la asignatura de computadoras en la enseñanza, en el Instituto Superior de Ciencias de Educación del Huambo. XIII Congresso Internacional de Pedagogía. La Habana, Cuba.

Traverso, Hugo Emilio, e outros. (2013). Herramientas de la Web 2.0 aplicadas a la educación. UNPL. Argentina. Disponível em: http://sedici.unlp.edu.ar/handle/10915/27532. Consulta: 05/07/2017

Vermelho, Sônia Cristina; Velho, Ana Paula Machado; Bertoncello, Valdecir. Sobre o conceito de redes sociais e seus pesquisadores. Educação e Pesquisa Volume. 41. Número. 4. Brasil (Pp. 863-881). 